\title{
The Coordinated Replenishment Policy in a decentralized Assembly System
}

\author{
Jianhong $\mathrm{Yu}^{1, *}$ \\ School of Business, Jianghan University, Wuhan, China
}

\begin{abstract}
The synchronization and coordination of material flows in the assembly system is much more important and complex. To analyze how to coordinate the material flows for the assembly supply chain, we consider an assembly system with two component-suppliers and one manufacturer, under stochastic finished product demand. Two replenishment policies are proposed and analyzed. One is the decentralized replenishment policy without horizontal coordination, and the other is the coordinated replenishment policy with horizontal coordination. Results show that there is a unique optimal solution to minimize the supply chain cost, and coordination replenishment policy can efficiently deduce supply chain cost due to the horizontal coordination.
\end{abstract}

Keywords-coordinated replenishment; assembly system; horizontal coordination

\section{INTRODUCTION}

The coordination of components is a key issue in assembly system [1]. In an assembly system, the finished product is assembled with hundreds or thousands components. Due to the complexity and difficulty of coordination, in practice, most manufacturers only consider the vertical coordination between suppliers and the manufacturer, but don't consider the horizontal coordination between suppliers. However, in assembly system, the quantity of final products is decided by the lest quantity of components. The discoordination between suppliers not only reduces the service level, but also increases the components' inventory cost. Therefore, how to realize the coordination between suppliers is very important.

Nowadays, some researchers have done some relative research. Khouja and Goyal [2] conduct a comprehensive review of coordinated replenishment literature from 1989 to 2005, which addresses constrained, stochastic, and dynamic demands. De Boeck and Vandaele [3] analyze the problem of synchronizing material flows, and find synchronization can reduce the overall pipeline inventory. Ma and Gong [4] set a production-distribution model, and propose to order parts replenishments proportionally between various suppliers. De Boeck and Vandaele [5] study a generic first-come first-serve assembly system, and find that parts supply has to be synchronised and needs a cap in order to shut down the input streams. Limère [6] analyzes a multi-component assembly system, and finds that hybrid materials feeding policies is preferred. Li et al. [7] propose a holding-cost subsidy contract to coordinate the decentralized assembly system. Most recently, Li et al. [8] investigated collaborative scheduling in the assembly system, and show that the performance of supply-hub coordinated schedule is superior to that of decision made independently by the manufacturer and the supplier.

From the above literatures, most researchers only discuss the collaboration of upstream materials in centralized decision model. Though some literatures discusses how to collaborate upstream materials of assembly system, there are still issues that need to be addressed. As we all know, the bill of material (BOM) is very important to the assembly system. A bad decision on one component's replenishment in a BOM will invalidate other components' optimal replenishment decisions, thus leading to low SC efficiency [9]. In this paper, we considers the horizontal coordination between suppliers and propose a simple and practical replenishment policy based on $(\mathrm{Q}, \mathrm{r})$ policy, which is popular used by many manufacturers in practice.

\section{Problem DEScRiption AND NotATIONS}

We consider a two-key-suppliers and one-manufacturer system, where in each supplier provides a different component. The manufacturer needs the two key parts to produce a final product. It is an assemble-to-order (ATO) system. The components are replenished according to the $(\mathrm{Q}, \mathrm{r})$ policy. Without loss of generality, the time for assembling the components is assumed to be negligible, which is appropriate when the suppliers are far from the manufacturer. In addition, we don't consider the residual value of surplus products and the assembly cost. Therefore, products are only assembled to order and no parts are sent to the manufacturer without orders from customers. The unmet demand is fully backordered.

We assume the demand of the finished product is stochastic, and the per unit time demand during lead time follows a normal distribution with mean $\mu$ and standard deviation $\sigma$. The two suppliers are reliable. Without loss of generality, we assume the lead time of component 1 is longer than that of component 2, and both suppliers have the same internal service level.

The inventory control policy is CVMI (Consignment Vendor Management Inventory), that is suppliers are in charge of the replenishment policy of components, and suppliers are only paid for the components used by the manufacturer. The manufacturer adopts a multi-period rolling plan and information sharing to coordinate material flows, that is, the vertical coordination between suppliers and manufacturer is realized. However, according to the bill of materials (BOM), to improve the performance of the whole system, not only the vertical coordination is needed, but also the horizontal coordination between suppliers is very necessary. The horizontal coordination can efficiently deduce the mismatching probability of 
components, and can lower the probability of backorders and increase customer service level.

In our model, suppliers bear the inventory holding cost, which consist of the average regular and safety inventory holding cost and backlogging inventory cost. In addition, the backlogging inventory cost is the inventory cost that caused by the stockout of other parts.

The notations are summarized in table 1.

TABLE I. THE DEFINITION OF NOTATIONS

\begin{tabular}{|c|c|c|c|}
\hline notation & definition & notation & definition \\
\hline$i$ & the number of component, $i=1,2$ & $f_{i}\left(x_{i}\right)$ & the density function of $x_{i}$ \\
\hline$L_{i}$ & replenishment lead time of component $i$ & $F_{i}\left(x_{i}\right)$ & the cumulative probability function of $x_{i}$, \\
\hline$h_{i}$ & the inventory cost per unit per time of component $i$ & $\mu_{i}$ & $\begin{array}{l}\text { the mean of demand for component } i \text { during lead time } L_{i} \text {, where } \\
\mu_{i}=\mu L_{i}\end{array}$ \\
\hline$F_{i}$ & $\begin{array}{l}\text { the fixed replenishment cost of components } i \text { per } \\
\text { replenishment cycle }\end{array}$ & $\sigma_{i}$ & $\begin{array}{l}\text { the standard deviation of demand for component } i \text { during lead time } \\
L_{i} \text {, where } \sigma_{i}=\sigma \sqrt{L}_{i}\end{array}$ \\
\hline$D$ & the expected value of annual demand & $z$ & the safety factor, $z>0$ \\
\hline$\pi$ & the penalty cost per unit backorder & $\Phi(z)$ & the internal service level of component $i$ \\
\hline$x_{i}$ & the lead time demand of component $i$ & $P$ & the customer service level for the manufacturer \\
\hline$Q_{i}$ & the replenishment quantity of part $i$ & $P^{0}$ & the lowest limited service level, that is $P^{0} \leq P$ \\
\hline$r_{i}$ & the reorder point of component $i$ & $z^{0}$ & the value of safety factor when $P=P^{0}$ \\
\hline
\end{tabular}

\section{THE DECENTRALIZED REPLENISHMENT POLICY WITHOUT HORIZONTAL COORDINATION}

In this replenishment policy, according to manufacturer's demand planning and service level requirement, the suppliers make decisions on component replenishment plans to minimize its cost. The horizontal coordination between suppliers is not considered, which may result in excess inventory of one part type, while the other component is out of stock.

The probability of component ${ }^{i}$ out of stock during the lead time is $P\left\{x_{i}>r_{i}\right\}=\int_{r_{i}}^{+\infty} f_{i}\left(x_{i}\right) d x_{i}=1-\Phi(z)$, where $\Phi(z)$ is the internal service level of component ${ }^{i}$. When $\Phi(z)$ is high, such as $\Phi(z) \in(0.90,0.99)$, the probability of having both components shortages simultaneously is very small and negligible [10]. The expected backorder per cycle can be expressed as $B_{i}\left(r_{i}\right)=\int_{r_{i}}^{\infty}\left(x_{i}-r_{i}\right) f\left(x_{i}\right) d x_{i}$. So, the expected cost of supplier $i$ per unit time can be expressed as:

$$
E\left[T C_{s i}\right]=\frac{D}{Q_{i}} F_{i}+h_{i}\left(\frac{Q_{i}}{2}+z \sigma \sqrt{L_{i}}\right)+h_{i} \frac{D}{Q_{j}} \int_{r_{j}}^{+\infty}\left(x_{j}-r_{j}\right) f_{j}\left(x_{j}\right) d x_{j}
$$

Where the first term is the fixed replenishment cost per unit time of supplier ${ }^{i}$; the second term is the regular and safety inventory cost per unit time of supplier $i$; while the third term is the backlogging inventory cost per unit time of supplier ${ }^{i}$ due to lack of component $j,(j=1,2, j \neq i)$. Note that backlogging is caused by the shortage of the components.

Management requires customer service level satisfies $P=\Phi(z)^{2} \geq P^{0}=\Phi\left(z^{0}\right)^{2}$, that is $\Phi(z) \geq \Phi\left(z^{0}\right)$.

The expected cost of the manufacturer per unit time can be expressed as:

$$
E\left[T C_{m}\right]=\pi \sum_{i=1}^{2} \frac{D}{Q_{i}} \int_{r_{i}}^{\infty}\left(x_{i}-r_{i}\right) f_{i}\left(x_{i}\right) d x_{i}
$$

The expected cost of the whole supply chain per unit time $E\left[T C_{s c}\right]=E\left[T C_{s i}\right]+E\left[T C_{m}\right]$.

\section{A. Proposition 1}

In the decentralized replenishment policy without horizontal coordination, the expected cost per unit time of supplier ${ }^{i}$ is convex in $Q_{i}$, and there is a unique optimal quantity $Q_{i}^{*}$ that minimizes $E\left[T C_{s i}\right]$ and $z=z^{0}$. Therefore, the optimal quantity can be characterized as:

$$
Q_{i}^{*}=\sqrt{2 D F_{i} / h_{i}}
$$

Proof: Due to lacking of horizontal coordination, the expect cost of supplier $i$ is strictly increasing in $z$, so there is $z=z^{0}$. Obviously, the expect cost of supplier ${ }^{i}$ is strictly convex in $Q$, and we get the optimal value from the first order condition.

\section{THE COORDINATED REPLENISHMENT POLICY WITH HORIZONTAL COORDINATION}

In this paper, we use the common replenishment cycle to coordinate different components' replenishment, in order to optimize the total cost and improve service level. To simplify, a base replenishment quantity is used to instead of the common replenishment cycle. Let $Q$ be the base replenishment quantity. Without loss of generality, let $Q$ be the smaller quantity of component, and let $k Q$ be the replenishment quantity of supplier 2, where $k$ is an integer.

The customer service level under The coordinated replenishment policy with horizontal coordination is limited to the lowest internal service level, so $P$ satisfies $P=\Phi(z) \geq \Phi\left(z^{0}\right)$.

The expected supply chain cost per unit time can thus be expressed as: 


$$
\begin{aligned}
E\left[T C_{s c}\right]= & \frac{D}{Q} F_{1}+h_{1}\left(\frac{Q}{2}+z \sigma \sqrt{L_{1}}\right)+\frac{D}{k Q} h_{1}\left[\int_{\mu L_{1}+z \sigma \sqrt{L_{2}}}^{r_{1}}\left(x_{1}-\mu L_{1}-z \sigma \sqrt{L_{2}}\right) f_{1}\left(x_{1}\right) d x_{1}\right. \\
& \left.+\int_{r_{1}}^{\infty}\left(z \sigma\left(\sqrt{L_{1}}-\sqrt{L_{2}}\right) f_{1}\left(x_{1}\right) d x_{1}\right)\right]+\frac{D}{k Q} F_{2}+h_{2}\left(\frac{k Q}{2}+z \sigma \sqrt{L_{2}}\right) \\
& +h_{2} \frac{D}{k Q}(k-1) \int_{r_{1}}^{\infty}\left(x_{1}-r_{1}\right) f_{1}\left(x_{1}\right) d x_{1}+\pi \frac{D}{k Q}\left[(k-1) \int_{r_{1}}^{\infty}\left(x_{1}-r_{1}\right) f_{1}\left(x_{1}\right) d x_{1}\right. \\
& \left.+\int_{\mu L_{1}+z \sigma \sqrt{L_{2}}}^{\infty}\left[x_{1}-\left(\mu L_{1}+z \sigma \sqrt{L_{2}}\right)\right] f_{1}\left(x_{1}\right) d x_{1}\right]
\end{aligned}
$$

Where the first three terms are respectively the replenishment cost, the regular and safety inventory cost, and the backlogging inventory cost of component 1 ; while the 4-6 terms are those of component 2, the 7th term is the backorder cost per unit time. To simplify the above function, let

$$
\begin{aligned}
& B=F_{2}-\left(h_{2}+\pi\right) \int_{r_{1}}^{\infty}\left(x_{1}-r_{1}\right) f_{1}\left(x_{1}\right) d x_{1}+\pi \int_{\mu L_{1}+2 \sigma \sqrt{L_{2}}}^{\infty}\left[x_{1}-\left(\mu L_{1}+2 \sigma \sqrt{L_{2}}\right)\right] f_{1}\left(x_{1}\right) d x_{1} \\
& \left.\quad+h_{1} \iint_{\mu L_{1}+2 \sigma \sqrt{L_{2}}}^{r_{1}}\left(x_{1}-\mu L_{1}-2 \sigma \sqrt{L_{2}}\right) f_{1}\left(x_{1}\right) d x_{1}+\int_{r_{1}}^{\infty}\left(2 \sigma\left(\sqrt{L_{1}}-\sqrt{L_{2}}\right) f_{1}\left(x_{1}\right) d x_{1}\right)\right], \\
& \text { and } A=F_{1}+\left(h_{2}+\pi\right) \int_{r_{1}}^{\infty}\left(x_{1}-r_{1}\right) f_{1}\left(x_{1}\right) d x_{1} .
\end{aligned},
$$

The expected supply chain cost can be reformed as,

$$
E\left[T C_{s c}\right]=\frac{D}{Q} A+\frac{h_{1}}{2} Q+\frac{D}{k Q} B+\frac{h_{2}}{2} k Q+\left(h_{1} \sqrt{L_{1}}+h_{2} \sqrt{L_{2}}\right) z \sigma
$$

To obtain the following Proposition, we need to relax the integral constraint of $k$.

\section{A. Proposition 2}

There exists a unique optimal solution that minimizes the expected supply chain cost, when $B>0$. For any given $z$, $E\left[T C_{s c}\right]$ is a joint convex function of $Q$ and $k$, and the optimal value can be denoted as

$$
Q^{*}=\sqrt{\frac{2 D A}{h_{1}}} \quad \alpha v \delta k^{*}=\sqrt{\frac{B h_{1}}{A h_{2}}}
$$

Proof: Relaxing the integral constraint of $k$, we can get the derivative of $E\left[T C_{s c}\right]$ with respect to $z, Q$ and $k$,

$$
\begin{gathered}
\frac{\partial E\left[T C_{s c]}\right]}{\partial z}=\sigma\left(h_{1} \sqrt{L_{1}}+h_{2} \sqrt{L_{2}}\right)-\frac{(k-1) D}{k Q}\left(h_{2}+\pi\right) \sigma \sqrt{L_{1}} \int_{r_{1}}^{\infty} f_{1}\left(x_{1}\right) d x_{1} \\
-\frac{D}{k Q} \pi \sigma \sqrt{L_{2}} \int_{\mu L_{1}+z \sigma \sqrt{L_{2}}}^{\infty} f_{1}\left(x_{1}\right) d x_{1} \\
\frac{\partial^{2} E\left[T C_{s c}\right]}{\partial z^{2}}=\frac{(k-1) D}{k Q}\left(h_{2}+\pi\right) \sigma^{2} L_{1} f_{1}\left(r_{1}\right)+\frac{D}{k Q} \pi \sigma^{2} L_{2} f_{1}\left(\mu L_{1}+z \sigma \sqrt{L_{2}}\right)>0 \\
\frac{\partial E\left[T C_{s c}\right]}{\partial Q}=-\frac{D}{Q^{2}}\left(A+\frac{B}{k}\right)+\frac{h_{1}+k h_{2}}{2} \\
\frac{\partial^{2} E[T C]}{\partial Q^{2}}=\frac{2 D}{Q^{3}}\left(A+\frac{B}{k}\right)>0 ; \frac{\partial E\left[T C_{s c}\right]}{\partial k}=-\frac{D B}{k^{2} Q}+\frac{h_{2} Q}{2} \\
\frac{\partial^{2} E[T C]}{\partial k^{2}}=\frac{2 D B}{k^{3} Q}>0
\end{gathered}
$$

It is obviously that $\lim _{z \rightarrow \infty} \frac{\partial T C_{s c}}{\partial z}=\sigma\left(h_{1} \sqrt{L_{1}}+h_{2} \sqrt{L_{2}}\right)>0$ and $z>0$ 。 Therefore, there is a unique optimal solution $(Q, k, z)$ to minimize the supply chain cost per unit time.
For any giving ${ }^{z}$, it is easy to know that $E\left[T C_{s c}\right]$ is strictly convex in $Q$ for any giving $k>0$, and it is also strictly convex in $k$ for any giving $Q>0$. To prove $E\left[T C_{s c}\right]$ is joint strictly in $Q$ and $k$, we get the optimal value $Q=\sqrt{\frac{2 D\left(A+\frac{B}{k}\right)}{h_{1}+k h_{2}}} \quad$ and $k=\sqrt{\frac{2 D B}{h_{2} Q^{2}}}$. Hessian matrix about $Q$ and $k$ of $E\left[T C_{s c}\right]$, which satisfies the equation $H=\frac{4 D^{2}}{k^{4} Q^{4}}\left(k A B+B^{2}\right)-\left(\frac{D B}{k^{2} Q^{2}}+\frac{h_{2}}{2}\right)=\frac{k A}{B} h_{2}^{2}>0$

Therefore, $E\left[T C_{s c}\right]$ is joint convex in $Q$ and $k$, and the optimal values satisfy the first order condition.

Substituting (2) and (3) into (1), we can obtain the optimal supply chain cost,

$$
E\left[T C_{s c}\right]=\sqrt{2 D A h_{1}}+\sqrt{2 D B h_{2}}+\left(h_{1} \sqrt{L_{1}}+h_{2} \sqrt{L_{2}}\right) z \sigma_{(7)}
$$

\section{NUMERICAL ANALYSIS}

To further analyze the coordinated replenishment policy with horizontal coordination and the effect parameters, let $D=5500, \mu=15, \sigma=10, L_{1}=25$ days, $L_{2}=20$ days, $h_{1}=35, h_{2}=25, F_{1}=100, F_{2}=350, \pi=80$, and $\Phi\left(z^{0}\right) \geq 95 \%$. Let superscript ${ }^{d}$ and ${ }^{c}$ respectively denote the decentralized replenishment policy without horizontal coordination and the coordinated replenishment policy with horizontal coordination.

The results of effect of service level are shown in Figure 2. The left picture of Figure 2 analyzes the effect of internal service level $\Phi(z)$ on the supply chain cost. Let $\Phi(z)$ range from $90 \%$ to $99.99 \%$, and find that supply chain costs of both strategies first decrease with $\Phi(z)$ and then increase. However, the inflection points of the two cost curves are different, and that of the coordinated replenishment policy is much lower than that of the decentralized replenishment policy. The right picture of Figure 2 shows the impacts of $P^{0}$ are different from $\Phi(z)$. It shows that a low $P^{0}$ has not any effect on the supply chain cost of the coordinated replenishment policy. However, when $P^{0}$ is higher enough, the cost curves will significantly go up. The effect of $P^{0}$ on the decentralized replenishment policy is similar to that of $\Phi(z)$, due to $\Phi(z)^{2}=P$. Obviously that the coordinated replenishment policy always dominates the other policy, regardless of the service level. 


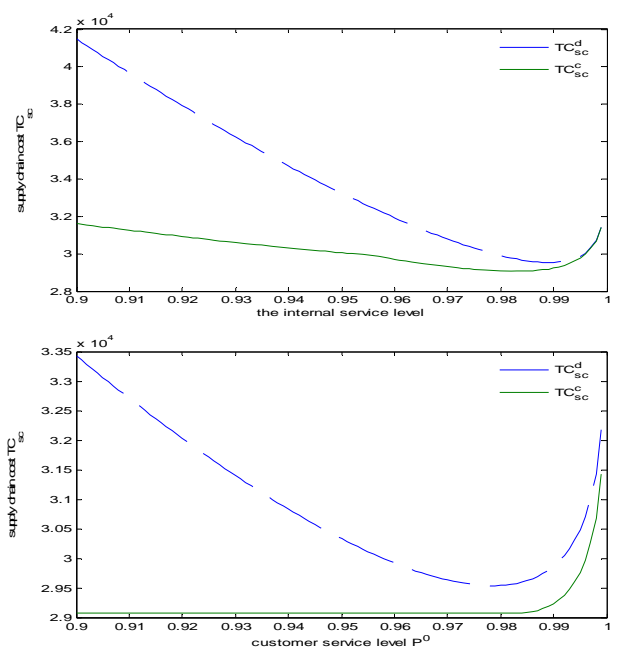

FIGURE I. THE EFFECT OF SERVICE LEVEL

The results of parameters' effects are shown in Table 2, where the base data means the results are calculated based on the data in the above section, and $\Delta T C_{s c}=\frac{T C_{s c}^{d}-T C_{s c}^{c}}{T C_{s c}^{d}} * 100 \%$. Obviously, the customer service level under the decentralized policy always satisfies $P=\Phi\left(z^{0}\right)^{2}=90.25 \%$.

TABLE II. THE IMPACTS OF PARAMETERS ON BOTH POLICIES

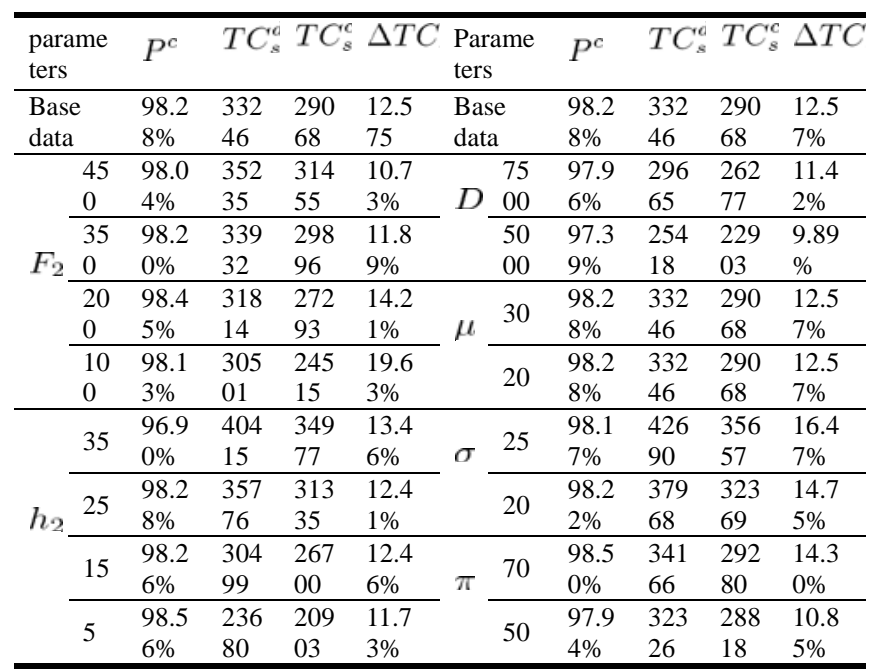

Table 2 shows that when the parameters decrease, the expected supply chain cost of both policies will decrease. Customer service levels of coordinated policy increases with $D$ and $\pi$, and decrease with $\sigma$. The cost advantage of coordinated policy increases with $h_{2}$ and $\sigma$, and decreases with $h_{2}$ and $\pi$, meanwhile there is no clear relationship between cost and $D$. However, coordinated policy still outperforms the decentralized policy, even when $\pi$ is much larger. Results also show that component parameters have different effects on customer service level. $P^{c}$ increases as $F_{2}$ and $h_{2}$ decrease, but only when they vary within a certain range.

\section{CONCLUSION}

In assembly systems, shortage of one or several components may discontinue the whole production process and result in huge loss. Coordination and synchronization of material flows is very important for improving supply chain efficiency. This paper studies the coordination replenishment policies in a decentralized assembly system. We propose and compare two different policies, which are the decentralized replenishment policy without horizontal coordination and the coordination replenishment policy with horizontal coordination. The results show that, (1) There is a unique optimal solution in the both replenishment policies. (2) Based on the parameters analysis, the coordination replenishment policy with horizontal coordination has obviously cost advantage than the decentralized replenishment policy without horizontal coordination. (3) The customer service level $P^{0}$ can affect the supply chain cost of the decentralized replenishment policy, but it has no impact on that of the coordinated replenishment policy when it is lower than a certain value.

\section{ACKNOWLEDGEMENT}

This research was financially supported by Hubei Provincial Department of Education Humanities and social sciences research project (17G058), the Natural Sciences Foundation of China under Grants NSFC71471084, the key open-ended program of Wuhan Research Institute of Jianghan University (IWHS2016104), and the Funding under the Project of "Superiority Characteristic Disciplines Group of Hubei Provincial Higher Universities-Urban Circle Economy and Industrial Integration Management”.

\section{REFERENCES}

[1] J. S. Song, P. Zipkin. Supply chain operations: assemble-to-order systems. Handbooks in Operation Research and Management Science, 11. Supply Chain Management. Elsevier, Amsterdam, The Netherlands. (2003)

[2] M. Khouja, S. Goyal. A review of the joint replenishment problem literature: 1989-2005. Eur. J. Oper. Res. 186(2008) 1-16.

[3] L. D. Boeck, N. Vandaele.. Coordination and synchronization of material flows in supply chains: an analytical approach. Int. J. Prod. Econ. 116(2008) 119-207.

[4] S. H. Ma, F. M. Gong. Collaborative decision of distribution lot-sizing among suppliers based on supply-hub. Industrial Engineering and Management, 14(2009) 1-9.

[5] L. D. Boeck, N.Vandaele. Analytical analysis of a generic assembly system. Int. J. Prod. Econ. 131(2011) 107-114.

[6] V. Limère. To kit or not to kit: optimizing part feeding in the automotive assembly industry.4OR-Q. J. Oper. Res. 11(2013) 97-98.

[7] S. Y. Li, D. Z. Zhang, F. P. Jin. Base inventory cooperation strategy of multi-parts with supply-hub. International Journal of Business and Management, 8(2013) 96-104.

[8] G. Li, F. Lv, X. Guan. Collaborative scheduling model for supply-hub with multiple suppliers and multiple manufacturers. The Scientific World Journal, http://dx.doi.org/10.1155/2014/894573, (2014).

[9] O. Fujiwara, D. Swdarage. An optimal $(Q, r)$ policy for a multipart assembly system under stochastic part procurement lead times[J]. Eur. J. Oper. Res. 100(1997) 550-556.

[10] H. Gurnani, R. Akella, J. Lehoczky. Supply management in assembly systems with random yield and random demand[J]. IIE Transaction, 32(2000) 701-714. 